\title{
Physical Interference Driven Dynamic Spectrum Management
}

\author{
Lei Yang, Lili Cao and Heather Zheng \\ Department of Computer Science, University of California, Santa Barbara, CA 93106 \\ leiyang, lilicao, htzheng@cs.ucsb.edu
}

\begin{abstract}
Dynamic spectrum management can drastically improve the performance of wireless networks struggling under increasing user demands. However, performing efficient spectrum allocation is a complex and difficult process. Current proposals make the problem tractable by simplifying interference constraints as conflict graphs, but they face potential performance degradation from inaccurate interference estimation. In this paper, we show that conflict graphs, if optimized properly, can produce spectrum allocations that closely match those derived from the physical interference model. Thus we propose PLAN, a systematic framework to produce conflict graphs based on physical interference characteristics. PLAN first applies an analytical framework to derive the criterion for identifying conflicting neighbors, capturing the cumulative effect of interference. PLAN then applies a local conflict adjustment algorithm to address heterogeneous interference conditions and improve spectrum allocation efficiency. Through detailed analysis and experimental evaluations, we show that PLAN builds a conflict graph to effectively represent the complex interference conditions and allow the reuse of efficient graph-based spectrum allocation solutions. PLAN also significantly outperforms the conventional graph model based solutions.
\end{abstract}

\section{INTRODUCTION}

Being a finite and scarce resource, spectrum must be managed efficiently to enable continuous growth of wireless networks and technologies. Managing spectrum, however, is highly challenging because it must address complex radio interference. A transmission succeeds only if the received signal strength divided by the total interference strength plus the noise (SINR) is above some threshold. When evaluating the quality of spectrum usage at any single transmission, one must consider the cumulative interference from other competing transmissions. Thus significant complexity is required to optimize the spectrum allocation.

Prior work on spectrum management simplifies this problem by assuming radio interference can be modeled by a conflict graph [5], [11], [14], [19], [21], [22]. The effect of interference is abstracted into pairwise binary metrics between transmissions. Two transmissions either conflict when using the same spectrum channel, or can use the same channel concurrently. Under this simple model, existing works have developed efficient spectrum allocation solutions.

On the other hand, recent works have shown that the use of conflict graph based interference models, could lead to large performance degradation in wireless networks [4], [12]. In its current design, the model fails to capture the cumulative effect of interference. Simultaneous activation of multiple links can cause enough cumulated interference to disrupt a transmission even though none of these links alone is harmful for the transmission. As a result, channel allocation under this model can lead to unnecessary conflicts or under-utilization of resources.

In this paper, we study the use of conflict graphs in the context of spectrum management. Interestingly, we conclude that the problem of graph based interference models lies in the way that the conflict graph is generated. We show that the conflict graph, if optimized judiciously, can produce spectrum management solutions that closely match those derived from the physical interference models.

Motivated by these observations, we propose Physical confLict grAph geNerator (PLAN), a systematic framework to distribute spectrum efficiently. PLAN builds the framework by combining a well-defined conflict graph generator with any (new or existing) graph-based spectrum allocation algorithm. PLAN makes an important contribution of capturing the cumulative effect of interference into the criterion for determining conflicting peers in the conflict graph. To do so, PLAN applies a two-step approach. First, assuming interference conditions are uniform across the network, PLAN develops an analytical framework to determine the optimal criterion for conflict detection, and uses such criterion to build a basic form of the conflict graph from the measured interference metrics. Second, extending to scenarios with heterogeneous interference conditions, PLAN introduces a local search algorithm to iteratively refine the conflict detection and adjust the spectrum allocations. In addition to accounting for the impact of physical environments, PLAN also considers the characteristics of the spectrum allocation algorithm.

We evaluate the performance of PLAN using both analytical and experimental results, which reveal the following findings:

- The performance of spectrum management solutions is highly sensitive to the choice of conflict graphs, particularly the criterion used to detect conflict peers. In our experiments, a small deviation in the criterion $(7 \mathrm{~m}$ in terms of the conflict distance) leads to a significant $45 \%$ performance degradation. This observation verifies the critical need of a good conflict graph generator.

- The optimal criterion of conflict depends heavily on several factors, including the allocation algorithm, the network topology, the receiver sensitivity as well as the radio propagation exponent. 


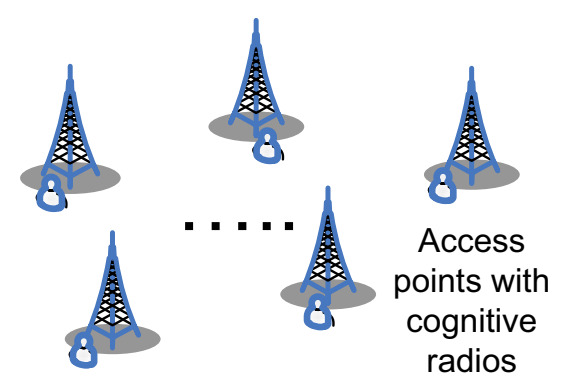

Fig. 1. An example scenario of spectrum management. Wireless access points access (and share) spectrum to connect their subscribers. Depending on their interference conditions, some access points can use the same spectrum channel concurrently, while others cannot.

- Under uniform interference conditions, the criterion of conflict peers is uniform across the network. PLAN's conflict graph generator offers similar performance as those of the optimal criterion derived from exhaustive search.

- Under heterogeneous interference conditions, PLAN refines the above mentioned uniform criterion at individual transmissions using iterative local adjustments. This adjustment results in 10-15\% improvement over the optimal uniform criterion, and performs comparably to the optimal allocation derived directly from the physical interference model. While being computational-efficient, PLAN leads to $<20 \%$ degradation in the spectrum utilization.

\section{BACKGROUND: INTERFERENCE-DRIVEN SPECTRUM ALLOCATION}

As background, we start from presenting the problem model of spectrum allocation. We show that the cumulative effect of interference makes the problem of spectrum allocation extremely challenging. We then briefly introduce the wellknown simplification via conflict graph based interference modeling, and identify the problems of such simplification.

\section{A. Problem Model}

As shown in Fig. 1, we consider a network of $L$ access points(AP) who obtain spectrum to serve their associated users. Throughout the paper, the notation of nodes and APs are interchangeable. Without loss of generality, we assume each node only serves one user*. For simplicity, we only consider the downlink traffic, i.e. the transmission from the node to the user. We assume that the available spectrum consists of $M$ non-overlapping channels. The channels are homogeneous, offering the same bandwidth and experiencing the same radio propagation characteristics.

Different from the conventional multi-channel multi-radio APs in mesh networks, nodes in our system are equipped with cognitive radios. They can access a flexible (rather than

\footnotetext{
*Extensions to multiple users can be done by expanding a node into multiple nodes, each representing a connection between the AP and a user. These new nodes all conflict with each other.
}

fixed) number of non-consecutive channels simultaneously. We represent the spectrum usage of node $n$ on channel $m$ as $a_{m, n}$ :

$$
a_{m, n}= \begin{cases}1, & \text { channel } m \text { is assigned to node } n \\ 0, & \text { otherwise. }\end{cases}
$$

Let the binary matrix $\mathbb{A}=\left\{a_{m, n}\right\}_{M \times L}$ represent the result of a spectrum allocation.

To model the impact of interference, we use a SINR-based receiver model where a node $i$ can successfully transmit to its receiving user at a predefined rate $\omega$ if and only if the user's received SINR is above a certain (rate-dependent) threshold $\beta$. For simplicity, we assume every node transmits at the same rate $\omega$. Let $b_{m, n}$ represent the normalized throughput between node $n$ and its user when it transmits via channel $m$ :

$$
b_{m, n}= \begin{cases}1, & \text { if } \frac{P_{i} / d_{i}^{\alpha}}{\sum_{\left\{j \mid a_{m, j}=1\right\}} P_{j} / d_{j, i}^{\alpha}+N} \geq \beta, \\ 0, & \text { otherwise. }\end{cases}
$$

where $P_{i}$ is the transmit power at node $i, d_{i}$ is the distance between node $i$ and its user, $d_{j, i}$ is the distance between any other node $j$ and the user of node $i, N$ is the noise power. This definition maps to the well-known physical interference model [7].

The goal of spectrum allocation is to maximize a system utility function based on the achieved throughput for each node-user pair $i$. Define the achieved throughput for each node-user pair $i$ as $A_{i}=\sum_{m=1}^{M} a_{m, i} \cdot b_{m, i}$. The spectrum allocation problem can be defined as:

$$
\begin{array}{cl}
\text { Find } & \mathbb{A}=\left\{a_{m, n}\right\}_{1 \leq n \leq L, 1 \leq m \leq M} \\
\text { Maximize } & \sum_{n=1}^{L} \mathbb{U}\left(A_{n}\right)=\sum_{n=1}^{L} \mathbb{U}\left(\sum_{m=1}^{M} a_{m, n} \cdot b_{m, n}\right)
\end{array}
$$

where $\mathbb{U}(\cdot)$ defines the system utility that the allocation intends to maximize. Example utility functions are (i) total spectrum utilization, $\mathbb{U}(x)=x$, (ii) proportional fairness, $\mathbb{U}(x)=$ $\log x$ [22].

The optimization problem (2) is highly complex because the effect of radio interference is accumulative and globalized. The throughput at each node $A_{n}$ depends on the channel usage of all other nodes in the network, regardless of their location.

\section{B. Conflict-Graph Based Simplification}

Prior work in this area simplifies the problem using a conflict-graph based interference model, or the so-called protocol model [8]. This model reduces the accumulative interference conditions into a set of pairwise interference constraints. Any two transmissions either conflict and cannot use the same channels concurrently or they do not conflict and can reuse the same channel. A graphic interpretation is to represent each (unidirectional) transmission as a vertex, and any two vertices are connected with an edge if the two transmissions interfere with each other. 
Formally, we represent the conflict graph model with an $L$ by $L$ binary matrix $\mathbb{C}=\left\{c_{n, k} \mid c_{n, k} \in\{0,1\}\right\}_{L \times L}$,

$c_{n, k}=\left\{\begin{array}{l}1, \quad \text { nodes } n \text { and } k \text { conflict with each other } \\ 0, \quad \text { nodes } n \text { and } k \text { can reuse the same channel. }\end{array}\right.$

A node $k$ is a neighbor of node $n$ if $c_{n, k}=1$. Under this model, the throughput of a transmission on channel $m$ is

$$
b_{m, n}^{\prime}= \begin{cases}1, & \forall_{k}, a_{m, k} \cdot c_{n, k}=0 \\ 0, & \text { otherwise. }\end{cases}
$$

In other words, $b_{m, n}^{\prime}=1$ iff none of $n$ 's conflicting neighbors is assigned with channel $m$.

Under the conflict graph interference model, the problem of spectrum allocation becomes:

$$
\begin{array}{rc}
\text { Find } & \mathbb{A}=\left\{a_{m, n}\right\}_{1 \leq n \leq L, 1 \leq m \leq M} \\
\text { Maximize } & \sum_{n=1}^{N} \mathbb{U}\left(\sum_{m=1}^{M} a_{m, n} \cdot b_{m, n}^{\prime}\right)
\end{array}
$$

Under the pair-wise and local constraints defined by a conflict graph, conventional optimization techniques such as graph-coloring and local search can be applied to produce efficient solutions to (4). Prior works have developed both centralized and distributed solutions [5], [14], [16], [22].

\section{Deriving Conflict Graphs}

While extensive efforts have been devoted to developing efficient allocation algorithms under the graph interference model, little consideration is given to deriving a proper conflict graph. The most commonly used criterion is the interference range where any two transmissions conflict if any transmitter is within the interference range of the other link's receiver. The widely-used choice of the interference range is twice the communication range.

Recent efforts have developed measurement-based solutions to define conflict graphs. To determine whether two links conflict, they perform isolated measurements on the transmission quality when both links are on and when only one of them is on. In [13], two links are declared conflicting if the total throughput when both links are active is significantly lower than the total throughput when only one is active. The work in [15] improves the measurement scalability by developing theoretical models on RSSI measurements. Finally, a proactive probing approach was proposed by [1] to determine the quality of transmissions when both links transmit.

Under the physical interference model defined by (1), the equivalent approach to the above solutions is to perform the isolated pairwise measurements to compare the received signal strength to the interference signal strength. Two transmissions conflict if the corresponding interference signal is strong enough compared to the signal strength. That is,

$$
c_{i, j}=1 \text { iff } \frac{P_{i} / d_{i}^{\alpha}}{P_{j} / d_{i, j}^{\alpha}+N} \leq \phi_{i} \text { or } \frac{P_{j} / d_{j}^{\alpha}}{P_{i} / d_{j, i}^{\alpha}+N} \leq \phi_{j}
$$

where $\phi_{i}$ is an optimization parameter. Under the uniform transmit power $P_{i}=P$ and AP-user distance $d_{i}=d$, (5)

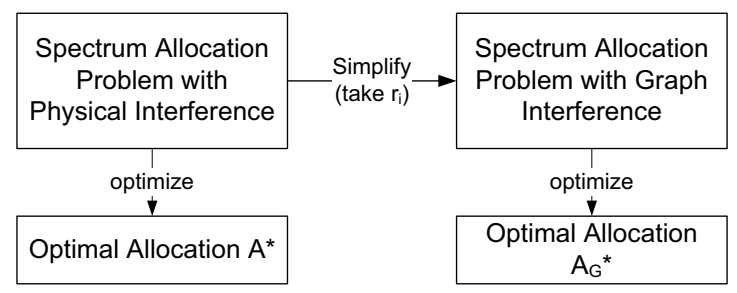

Fig. 2. The methodology of comparing the difference between the conflict graph model and the physical interference model.

reduces to a distance-driven threshold $r_{i}$ :

$$
\begin{aligned}
& c_{i, j}=1 \text { iff } d_{i, j}<\max \left\{r_{i}, r_{j}\right\}, \\
\text { where } \quad & r_{i}=\left(\frac{P}{P /\left(d^{\alpha} \phi_{i}\right)-N}\right)^{1 / \alpha} .
\end{aligned}
$$

\section{Challenges Facing the Graph based Interference Model}

While the use of the graph-based interference model greatly simplifies the spectrum allocation problem, it also faces significant challenges. In particular, the pairwise interference constraints ignore the effect of the accumulative interference - simultaneous activation of multiple links can cause enough cumulated interference to disrupt a transmission even though none of these links alone is harmful for the transmission. Without a proper graph design, the resulting channel allocation can lead to under-utilization of spectrum or unnecessary conflicts. Similar conclusions have been identified in the context of wireless capacity and channel scheduling [6], [7], [12].

\section{Conflict-Graph BASEd Spectrum Allocation: REVISITED}

Given the interesting performance/complexity tradeoff between the graph and physical interference models, a natural question arises: "Can we build a proper conflict graph so that it can capture the impact of the accumulative interference, and yet allows us to reuse the well-developed graph-based channel allocation algorithms to solve the spectrum management problem?"

To answer this question, we first quantify the performance difference between spectrum allocations derived from the physical interference model and the conflict graph model. Surprisingly, we found that by choosing individual $r_{i}^{\dagger}$ properly, we can always find the same optimal spectrum allocation (in the context of the physical interference model) using the conflict graph.

To do so, we use the methodology described by Fig. 2. For any instance of the spectrum allocation problem (2) under the physical interference model defined by (1), let the optimal allocation be $A^{*}$. By choosing a set of $r_{i}$, the same instance is converted into an instance under the graph model defined

\footnotetext{
${ }^{\dagger}$ This conclusion applies to the cases where each AP transmits at a fixed power $P$ and the AP-user distance is fixed to $d$. However, the same conclusion applies to cases where the power and distance are not uniform. In this case, the criterion of conflict is $\phi_{i}$ in (5).
} 
by (4). The optimal solution under the graph model is defined as $A_{G}^{*}$.

The following theorem shows that $A^{*}=A_{G}^{*}$.

Theorem 1: Assuming the utility function is the spectrum utilization with $\mathbb{U}(x)=x$, and given any instance of problem (2), there exists a set of $r_{i}$, such that the instance of the simplified problem (4) has exactly the same optimal allocation, i.e., $A^{*}=A_{G}^{*}$.

Proof: Given the linear utility function, we can rewrite the optimization objective in (2) as $\sum_{m=1}^{M} \sum_{n=1}^{L} a_{m, n} \cdot b_{m, n}$. Because the channels have the same interference characteristics and optimization goals, the problem reduces to a single channel optimization problem. In the remainder of the proof we assume there is only one channel, $M=1$.

When building the conflict graph, we select $r_{i}$ for each node $i$ based on $A_{1, i}^{*}$. That is, if $A_{1, i}^{*}=0$, set $r_{i}=W$ where $W$ is the maximum distance between any two nodes. If $A_{1, i}^{*}=$ $1, r_{i}=0$. To prove $A^{*}=A_{G}^{*}$, first notice that for any $i$ such that $A_{1, i}^{*}=0, i$ will conflict with all other nodes in the conflict graph since $r_{i}$ is sufficiently large. Therefore $A_{G 1, i}^{*}=$ 0 , because otherwise $A_{G}^{*}$ will have only one node allocated. Next, since any two nodes $i, j$ with $A_{1, i}^{*}=1$ and $A_{1, j}^{*}=1$ cannot share an edge in the conflict graph, all nodes with $A_{1, i}^{*}=1$ must be allocated in $A_{G}^{*}$ because of $A_{G}^{*}$ 's optimality. Therefore $A^{*}=A_{G}^{*}$.

We note that although Theorem 1 proves that conflict graphs can be optimized to produce the same optimal solution derived from the physical interference model, it does not provide any solution to optimizing the graph without the knowledge of the optimal allocation result.

\section{A. Physical Conflict Graph Generator (PLAN)}

The above observation motivates us to develop PLAN, a practical and yet systematic framework to optimize the conflict graph that can produce spectrum allocations close to the optimal solution. Our design methodology of PLAN comes from the following observations:

- Theorem 1 implies that $r_{i}$ should be non-uniform across nodes/links. However, finding the optimal non-uniform $r_{i}$ can be highly challenging.

- Extensive results show that under a uniform $r_{i}=r$, optimizing $r$ can lead to significant performance improvements. For example, Fig. 3 shows that the spectrum utilization is highly sensitive to the choice of $r$. As $r$ changes from $47 m$ to $40 m$, the utilization degrades by $45 \%$.

Based on these observations, PLAN takes a two-step approach. First assuming $r_{i}$ is uniform, we develop an analytical framework to determine the optimal uniform $r_{i}=r$. Second, we progressively adjust $r_{i}$ at individual nodes to further improve the spectrum allocation. We will describe the analytical framework in Section IV and the algorithm for the individual adjustments in Section V.

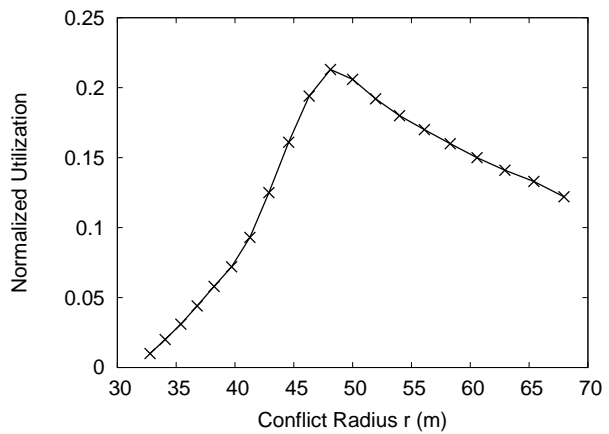

Fig. 3. The impact of $r$. We randomly deploy a set of nodes in the plane and use different values of $r$ to generate the conflict graph. We then use the allocation algorithm of [5] to generate the spectrum allocation, and evaluate the spectrum utilization defined by (2).

\section{An Analytical Framework for BUilding the CONFLICT GRAPHS}

In this section, we propose an analytical framework to produce the optimal conflict graph using uniform $r_{i}=r$. As a reference, we start from a worst-case analysis that derives the upper bounds on $r$. We then develop an allocation-aware approach to optimize $r$.

Our analysis makes the following assumptions. We assume that all the nodes are uniformly distributed in a circular disk with radius $R$, and the number of nodes $L \gg 1$. We assume each node has the same transmission power $P$ and each senderreceiver pair has the same distance $d$. Note that while these assumptions simplify the algorithm description, they do not limit the applicability of our analytical framework. We will discuss extensions to practical settings in Section VII.

\section{A. Worst-Case Analysis}

We start from a worst-case analysis to derive an upper bound on $r$. We compute the maximum level of interference a transmission can suffer by using $r_{i}=r$ to produce the conflict graph. We then use this measure to determine the $r$ required to guarantee that all the active transmissions are successful.

1) Multi-tier Interference: In the worst interference case, we know from geometry [17] that the nodes allocated with the same channel form a hexagonal topology shown in Fig. 4. When considering the interference produced by all the nodes, the center node's transmission will experience the heaviest interference from multiple tiers of neighbors. At tier $k$, there are $6 \cdot k$ nodes that are approximately $k \cdot r$ away from the center AP's receiver. We can estimate the interference as:

$$
\begin{aligned}
I(r)<\sum_{i=1}^{\lfloor R / r\rfloor} & 6 \cdot i \cdot \frac{P}{(i \cdot r)^{\alpha}}=\frac{6 P}{r^{\alpha}} \sum_{i=1}^{\lfloor R / r\rfloor} i^{1-\alpha} \\
\leq & \frac{6 P}{r^{\alpha}}\left(1+\int_{1}^{\lfloor R / r\rfloor} x^{1-\alpha} d x\right) \\
& =\frac{6 P}{r^{\alpha}}\left(1+\frac{\lfloor R / r\rfloor^{2-\alpha}-1}{2-\alpha}\right) .
\end{aligned}
$$




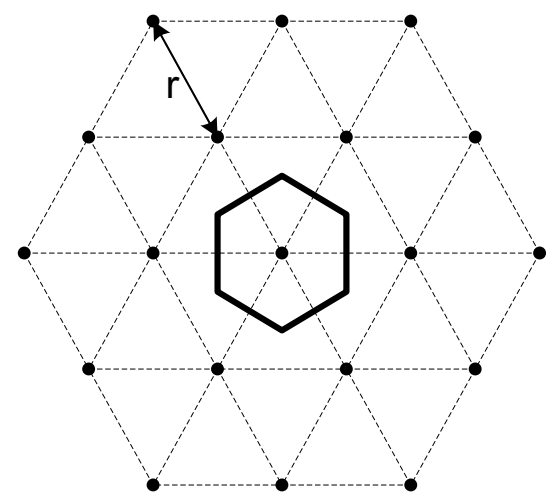

Fig. 4. The assignment when the center node suffers the most interference. All APs (represented by the solid points) on the hexagon are on the same channel.

In (7), the distance between the center AP's user and an AP in the $i$-th tier is approximated as $i \cdot r$ because we assume the AP-user distance $d$ is generally much smaller than the AP-AP distance.

To guarantee that the user receives packets successfully, we need:

$$
\frac{P / d^{\alpha}}{I(r)+N} \geq \beta
$$

The minimum $r$ that satisfies (8) is the required distance. Because the multi-tier interference metric over-estimates the interference, this maps to the upper bound $r_{u b}$ on $r$.

2) Single-tier Interference: When estimating the interference, previous literatures [9], [20] consider only the first tier of nodes because they produce the majority of the interference. In this case, the total interference only counts those of the 6 closest neighbors in Fig. 4:

$$
I(r)=6 \cdot \frac{P}{r^{\alpha}} .
$$

To satisfy (8), we get $r_{u b}=\frac{(6 P)^{1 / \alpha}}{\left(\frac{P}{\beta \cdot d \alpha}-N\right)^{1 / \alpha}}$.

To examine the accuracy of the above two bounds, we calculate the two upper bounds for $R=500 \mathrm{~m}$ and $d=5$ $\mathrm{m}$, using the same settings of Fig. 3. The bounds are $r_{u b}=$ $63.05 \mathrm{~m}$ for the multi-tier and $r_{u b}=38.73 \mathrm{~m}$ for the single-tier, respectively. Placing these values in Fig. 3, we see that the multi-tier estimation is overly conservative while the singletier estimation is overly aggressive by ignoring the interference from other tiers.

Intuitively, the multi-tier $r_{u b}$ is overly conservative because it does not consider the allocation efficiency. Note that under the conflict graph based interference model, the spectrum allocation problem is still NP-hard. Even with densely deployed APs, not all the nodes in the hexagonal topology will be allocated with the same channel. This observation motivates us to develop an allocation-aware algorithm to produce conflict graphs.

\section{B. Allocation-aware Conflict Graph Generation}

Different from the worst-case analysis, we derive the optimal $r$ from the average accumulative interference perceived

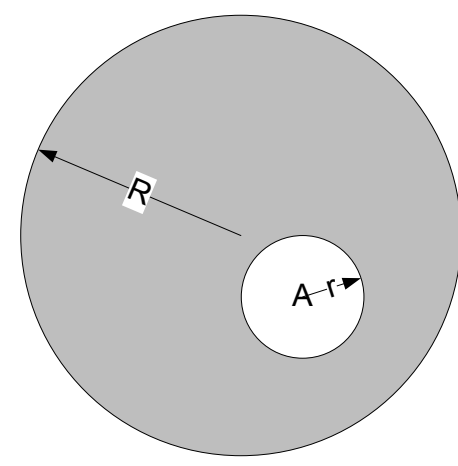

Fig. 5. A geometric map of the nodes that will generate interference to node A. The interference comes from the nodes in the gray region. $r$ is the conflict radius where nodes within $r$ distance from node A cannot use the same channel. $R$ is the radius of the network area.

by any AP-to-user transmission, while considering the effect of the allocation algorithm.

As shown in Fig 5, the interference received at node $A$ 's user comes from the nodes in the gray region outside the conflict circle defined by $r$. The aggregated interference level depends on the location of $A$, the conflict radius $r$ and the area radius $R$. It also depends on the node activation ratio $\mu$, i.e. the proportion of nodes in the grey area that are assigned with the same channel of $A . \mu$ is a function of $r$, and directly affects the spectrum utilization. Our goal is to choose $r$ appropriately such that the allocation algorithm can have a large active ratio $\mu$ yet every activated transmission has its received $S I N R \geq \beta$.

This can be formally written as:

$$
r^{*}=\min (r) \quad \text { s.t. } \quad \min _{1 \leq i \leq N} S I N R_{i}>\beta
$$

Intuitively, the optimal $r^{*}$ is limited by the minimum SINR of all active transmissions. In the circular topology, the center node's transmission receives the highest interference. Assuming the node ID of the central node is 1 , we have ${ }^{\ddagger}$ :

$$
E\left(I_{1}\right)=E\left(\sum_{i=2}^{N} I_{i 1}\right)=\int_{r}^{R} \frac{P}{x^{\alpha}} 2 \pi x \sigma \mu(r) d x
$$

The optimal conflict radius $r^{*}$ is the minimum $r$ where the expected SINR of node 1's transmission is no less than $\beta$, i.e.

$$
r^{*}=\min r \text { s.t. } E\left(I_{1}\right) \leq \frac{P}{\beta d^{\alpha}}-N \text {. }
$$

Clearly, the value of $r^{*}$ depends on $\mu(r)$. Next, we discuss how to estimate the algorithm-dependent $\mu(r)$.

\section{Deriving the Activation Ratio $\mu(r)$}

Deriving the exact form of $\mu(r)$ is hard given the randomness of the node location. Fortunately, our analytical results have derived two bounds on $\mu(r)$ which indicate that $\mu$ can be estimated by a allocation-dependent function of the average conflict degree $\bar{\Delta}$. Note that $\bar{\Delta}$ represents the average number

\footnotetext{
$\ddagger$ Because there are $L \gg 1$ nodes uniformly distributed in the large circular area, we use integration to estimate the accumulative interference from the grey region.
} 
of conflict neighbors per node. We also verify our findings through extensive simulations.

We first show two theorems on $\mu$.

Theorem 2: Given a conflict graph, we can achieve $\mu \geq$ $\frac{1}{\bar{\Delta}+1}$ using a simple greedy spectrum allocation algorithm.

Proof: The theorem follows directly from a theorem of Turán [18], which analyzes a simple greedy allocation algorithm as follows. "Starting from the allocation when all nodes are inactive, iteratively pick the node with smallest degree (if there are multiple nodes with this degree, take any one), make it active, then delete all its neighbors. Repeat until all nodes are active or deleted."

Turán [18] shows that the above algorithm can make at least $\frac{n}{\bar{\Delta}+1}$ active nodes, where $n$ is the total number of nodes in the input. This directly implies $\mu \geq \frac{1}{\bar{\Delta}+1}$.

Theorem 3: When nodes are uniformly distributed in a plane and $L \gg 1, \mu$ is upper bounded by $\frac{2 \pi / \sqrt{3}}{\bar{\Delta}+1}$, for any allocation algorithm.

Proof: When nodes have uniform distribution and $n$ is sufficiently large, the average degree is the number of nodes in a node's interference range:

$$
\bar{\Delta}=\sigma \pi r^{2}-1 .
$$

What is the maximum ratio of nodes that could be active simultaneously? Given the constraint that any two active nodes should be separated by at least $r$, finding the maximum number of active nodes is equivalent to finding the maximum number of circles with radius $r / 2$ that could be put on a plane without overlapping with each other. We know from geometry [17] that for the latter problem, the hexagonal placement (see Fig. 4) is the densest placement. Notice that each active node occupies a hexagon region (shown in Fig. 4) with edge length $\sqrt{3} r / 3$. The hexagon contains

$$
\sigma \cdot \frac{1}{2} \cdot \frac{\sqrt{3} r}{3} \cdot \frac{r}{2} \cdot 6=\frac{\sqrt{3}}{2} \sigma r^{2}
$$

number of nodes where $\sigma$ is the node density. The ratio is the reciprocal of (12). Combining (11) and (12), we get an upper bound of $\mu$ :

$$
\mu \leq \frac{1}{\frac{\sqrt{3}}{2} \sigma r^{2}}=\frac{2 \pi / \sqrt{3}}{\bar{\Delta}+1} .
$$

The results of Theorem 2 and 3 inspire us to approximate $\mu$ by $k / \bar{\Delta}$, where $1 \leq k \leq 2 \pi / \sqrt{3}$ is a constant that depends on the allocation algorithm. Assuming uniform node placements, $\bar{\Delta}+1$ can be estimated by $\pi r^{2} \sigma$, and the activation ratio can be represented by

$$
\mu(r)=k /\left(\pi r^{2} \sigma\right) .
$$

To verify $\mu(r)$, we use simulations to compare the actual active ratio to the estimated $\mu(r)$. Figure 6 shows the results using a local coordination based spectrum allocation algorithm [5]. We empirically find that $\mu(r) \approx 2 /\left(\pi r^{2} \sigma\right)$.

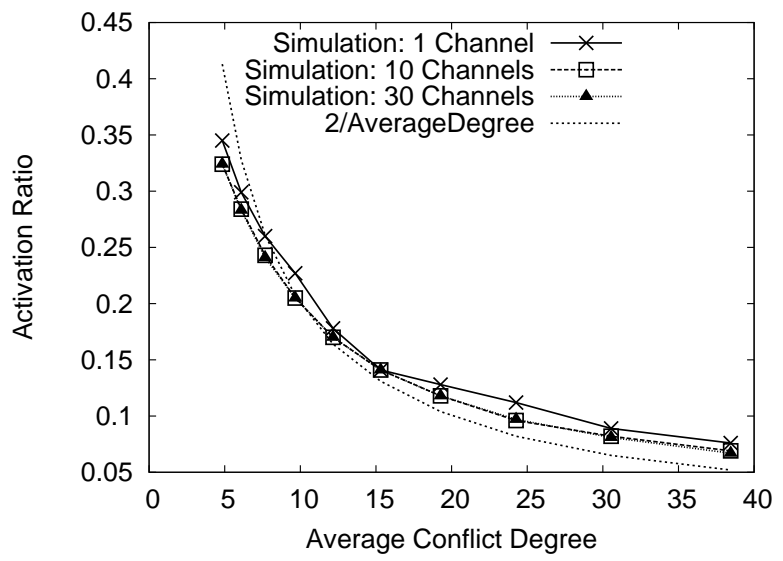

Fig. 6. The activation ratio as a function of the average conflict degree, using the local coordination based spectrum allocation algorithm [5]. For a fair comparison, we keep the same node deployment but vary $r$ to produce different conflict degree.

\section{Summary of Results}

Combining the results in the previous sections, we arrive at the following conclusions:

The Criterion to Generate the Conflict Graph: With the estimation of $\mu(r)$ in (13), we can solve $r^{*}$ as

$$
r^{*} \approx \begin{cases}R \exp \left(-\frac{1}{2} \mathrm{~W}\left(\frac{R^{2}}{k d^{2} \beta}\right)\right), & \alpha=2 \\ \left(\frac{2 k \beta}{\alpha-2}\right)^{1 / \alpha} \cdot d & \alpha>2, R \gg r\end{cases}
$$

where $\mathrm{W}$ is the Lambert $\mathrm{W}$ function (the inverse function of $\left.f(w)=w e^{w}\right)$. When $\alpha>2$, there is no closed form solution for $r^{*}$, but the above approximation for $R \gg r$. Because $r^{*}$ does not depend on the node density $\sigma$, individual nodes can derive $r^{*}$ directly from $\beta, d$ and $k$.

The Per-Channel Spectrum Utilization: Using $r^{*}$ and $\alpha>2$, we can also estimate the per-channel network throughput or the per-channel spectrum utilization as

$$
\begin{aligned}
U_{\text {thpt }}(\beta) & =\mu(r) \cdot T(\beta) \\
& =\frac{k}{\pi\left(r^{*}\right)^{2} \sigma} T(\beta) \\
& =\frac{k}{\pi \sigma}\left(\frac{\alpha-2}{2 k d^{2}}\right)^{1 / \alpha} \frac{T(\beta)}{\beta^{1 / \alpha}}
\end{aligned}
$$

where $T(\beta)$ represents the transmission rate when the decoding SINR threshold is $\beta$. For example, $T(\beta)=\log (1+\beta)$ when using a capacity achieving coding scheme. If $\beta$ is adjustable, we can also use (15) to derive the optimal $\beta$ that maximizes $U_{t h p t}$, and the corresponding $r^{*}$.

Some example values of our estimated $r^{*}$ in (7) as well as the upper bounds derived from the multi-tier and singletier interference estimations in Section IV-A are presented in Table I. We fix $\beta=10 d B$, set $\alpha=2$ or $\alpha=3$, and vary $R$ and $d$. We also include $r_{\text {opt }}$, the optimal uniform $r$ value obtained by the exhaustive search, as well as the degradation in spectrum utilization compared to the solution derived from the conflict graph produced with $r_{\text {opt }}$. We can see that our 
TABLE I

EXAMPLE VALUES OF $r^{*}, r_{u b}$, AND $r_{o p t}$, AS WELL AS THEIR PERFORMANCE DEGRADATION IN SPECTRUM UTILIZATION OVER THE SOLUTION USING A CONFLICT GRAPH PRODUCED FROM $r_{\text {opt }}$. ALL VALUES ARE IN METERS EXCEPT THE DEGRADATION VALUES.

\begin{tabular}{|c|c|c|c|c|c|c|}
\hline$\alpha$ & $R$ & $d$ & $r_{\text {opt }}$ & $\begin{array}{c}r^{*} \\
\text { (degradation) }\end{array}$ & $\begin{array}{c}r_{u b} \\
\text { single-tier } \\
\text { (degradation) }\end{array}$ & $\begin{array}{c}r_{u b} \\
\text { multi-tier } \\
\text { (degradation) }\end{array}$ \\
\hline 2 & 300 & 5 & 43.2 & $43.9(2 \%)$ & $38.7(35 \%)$ & $58.6(38 \%)$ \\
\hline 2 & 300 & 10 & 83.7 & $74.6(28 \%)$ & $77.4(20 \%)$ & $99.2(27 \%)$ \\
\hline 2 & 500 & 5 & 48.7 & $48.3(1.4 \%)$ & $38.7(70 \%)$ & $63.1(34 \%)$ \\
\hline 2 & 500 & 10 & 91.2 & $84.4(24 \%)$ & $77.5(58 \%)$ & $111.3(29 \%)$ \\
\hline 3 & 300 & 5 & 12.0 & $17.1(5.6 \%)$ & $19.6(15 \%)$ & $22.3(24 \%)$ \\
\hline 3 & 300 & 10 & 35.8 & $34.2(2.7 \%)$ & $39.1(9.2 \%)$ & $44.8(26 \%)$ \\
\hline 3 & 500 & 5 & 13.7 & $17.1(4.0 \%)$ & $19.6(12 \%)$ & $22.4(22 \%)$ \\
\hline 3 & 500 & 10 & 36.5 & $34.2(6.8 \%)$ & $39.1(8.7 \%)$ & $45.4(28 \%)$ \\
\hline
\end{tabular}

estimated $r^{*}$ performs comparably to the optimal $r$ in most cases, but suffers as $r_{\text {opt }}$ becomes large. This is because that the edge impact on the topology becomes more severe as $r$ increases. We also see that $r_{u b}$ from the multi-tier interference estimation is always too conservative. But those from singletier estimation are overly aggressive when $\alpha=2$ because it omits interference outside the first tier, but become overly conservative when $\alpha=3$ because it over-estimates the number of active nodes on the first tier.

\section{BEYOND UNIFORM $r$ : LOCAL CONFLICT AdJUSTMENT}

The analytical framework derives $r^{*}$ assuming idealized uniform interference conditions. In practice, nodes experience heterogeneous interference conditions because of the nonuniform node density, AP-user distance and transmit power. For example, measurements [10] show that APs are highly clustered, and their interference conditions vary significantly over locations.

To address such heterogeneity, PLAN introduces a local conflict adjustment algorithm to adjust $r_{i}$ based on local interference conditions. Starting from the $r^{*}$ derived from Section IV, PLAN first produces a conflict graph and determine the spectrum allocation accordingly. Next, based on their received SINR, nodes apply iterative adjustments to modify the conflict graph and the spectrum allocation. Intuitively, for any node $i$ with $\operatorname{SINR}<\beta$, PLAN increases its $r_{i}$ to add more conflict neighbors, reducing the level of interference. For any node $i$ with $\operatorname{SINR}>\beta$, PLAN decreases its $r_{i}$ to remove conflict neighbors, allowing more peers to reuse the channel and increase the spectrum utilization. Together, nodes apply these two adjustments iteratively based on the measured SINR to improve the usage of spectrum.

It should be noted that PLAN adjusts the conflict graph locally and thus the allocation algorithm can also adjust the spectrum allocation locally. These combined local adjustments avoid reapplying the allocation algorithm over the entire network, minimizing the computational overhead.

\section{A. Network-Aware Local Conflict Adjustment}

While the basic concept is simple, designing an effective local adjustment algorithm is challenging. Because interference is accumulative, adjustments at a single node could affect the received SINR of nodes across the network. For example, activating a node near a high SINR node could potentially reduce the SINR of multiple (far away) nodes to below $\beta$, leading to unnecessary performance degradation. Therefore, we must regulate the adjustments judiciously.

We propose a network-aware local adjustment algorithm which ranks local adjustments by their impact on network performance and chooses the best one iteratively. We first identify the node $n$ with the lowest SINR in the network. If $S I N R_{n}<\beta$, we find the node $n^{\prime}$ that uses the same channel and produces the largest interference to $n$, and increase the conflict radius of node $n$ to add a conflict edge between $n$ and $n^{\prime}$. We then modify the spectrum allocation based on the new conflict graph and evaluate the SINR again. We repeat the same procedure if the minimum SINR is still less than $\beta$. Next, when no node has SINR lower than $\beta$, we start to assign more channels to nodes. We rank nodes by their $\overline{S I N R}$. That is, for each node $i$, we calculate $\overline{S I N R}_{i}$ by averaging the SINR of $i$ on all of its allocated channels. After finding the node $n$ with the highest $\overline{S I N R}$, we reduce the conflict radius of $n$ to remove the conflict edge between $n$ and its farthest conflict neighbor and adapt the local channel allocation. The algorithm stops when no improvement to spectrum utilization is achieved in recent 10 adjustment attempts.

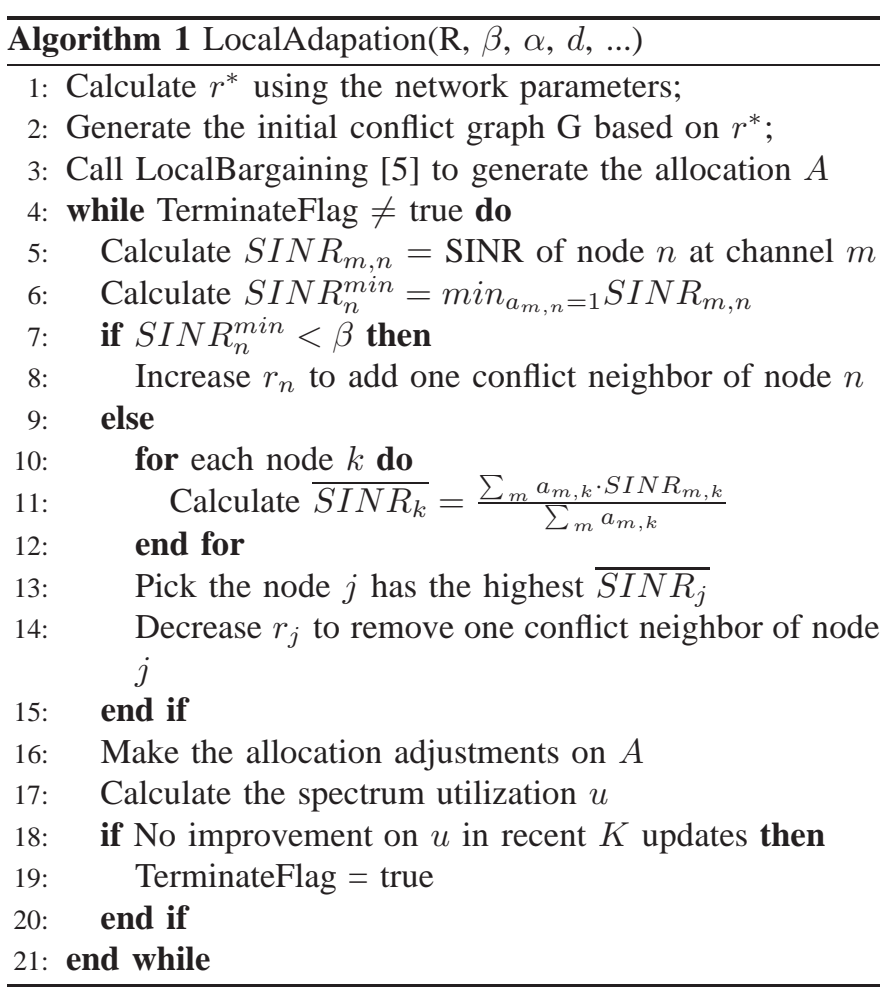

The detailed algorithm is shown in Algorithm 1. We note that the proposed local adjustment is a centralized greedy 
solution in order to consider the network-wide impact. Extensions to distributed solutions and practical protocols will be addressed in a future work.

\section{EXPERIMENTAL RESUlTS}

In this section, we perform network simulations to examine the performance of PLAN. We compare PLAN to other competitive conflict graph generation approaches, using the allocation algorithm proposed in [5] with the activation factor $k=2$. We also compare the spectrum allocation of PLAN to the optimal spectrum allocation derived directly from the physical interference model using the exhaustive search.

\section{A. Simulation Setup}

We place $L$ nodes (APs) and their users on a 2D plane. Each AP has one user with distance $d$ from it. We set $d$ to $5 \mathrm{~m}$ or $10 \mathrm{~m}$ in different simulations to represent the typical distance from WiFi users to APs. We set the transmission power of APs to $5 \mathrm{dBm}$ and the noise power to -102.5 $\mathrm{dBm}$. We calculate the signal and interference power using the signal propagation equation $P_{R X}=P_{T X} / d^{\alpha}$ with the signal propagation exponent $\alpha=2$ or 3 . To evaluate the allocation performance considering the accumulative interference, we calculate the SINR at each transmission and compare it with $\beta=10 d B$ : the receiver bit error rate is 0 if the SINR $\geq \beta$ and otherwise 1 . We assume $M=10$ channels. Table II summarizes the simulation parameters.

TABLE II

Simulation PARAMETERS

\begin{tabular}{|c|c|}
\hline Parameter & Value \\
\hline Propagation exponent $(\alpha)$ & 2 or 3 \\
\hline TX power $\left(P_{i}\right)$ & $5 \mathrm{dBm}$ \\
\hline Noise power $\left(N_{i}\right)$ & $-102.5 \mathrm{dBm}$ \\
\hline SNR threshold $(\beta)$ & $10 \mathrm{~dB}$ \\
\hline User to AP distance $(\mathrm{d})$ & $5 \mathrm{~m}$ or $10 \mathrm{~m}$ \\
\hline Number of Channels $(\mathrm{M})$ & 10 \\
\hline
\end{tabular}

To examine the impact of node placement, we use three types of network topologies.

- Uniform topology - The nodes are uniformly placed in a circular region of radius $R$. We divide the region into $L$ grid cells, each as a small square of length $D(30 \mathrm{~m}$ by default). We place one node randomly within each grid cell. This matches the settings of our analysis, and allow us to evaluate the analytically derived $r^{*}$.

- Clustered topology - The nodes are placed in a square region. We simulate a hotspot scenario by packing some nodes densely in a small area and other nodes randomly in the remaining area. We use this topology to examine the performance of PLAN in non-uniform networks.

- Trace-based topology - We deploy APs based on the measured AP location traces collected by PlaceLab [10].

We compare the spectrum allocation of four conflict graph generation methods, and the optimal allocation derived from the physical interference model.
1) UniCSV: The most conservative approach which uses the multi-tier worst-case analysis $r_{u b}$ to generate the conflict graph.

2) UniPLAN: The first step of PLAN, which uses the analytical conflict radius $r^{*}$ to generate the conflict graph.

3) UniOPT: The optimal uniform $r$ derived from the exhaustive search.

4) PLAN: The proposed conflict graph generation scheme using both the analytically derived $r^{*}$ and the local adjustment algorithm.

5) PhyOPT: The optimal spectrum allocation derived from the physical interference model. We perform an exponential search to examine all possible allocations and choose the best allocation that maximizes the spectrum utilization.

The performance metric is the normalized spectrum utilization $u$. Using $b_{i j} \in\{0,1\}$ to represent the normalized throughput of node $i$ at channel $j: b_{i j}=1$ if and only if node $i$ is allocated with channel $j$ and the corresponding SINR is higher than $\beta$. We have:

$$
u=\frac{\sum_{i=1}^{L} \sum_{j=1}^{M} b_{i j}}{M \cdot L}
$$

\section{B. Validating UniPLAN}

We start from uniform topologies. Fig. 7 compares UniPLAN with UniOPT and UniCSV for both $\alpha=2$ and 3 . We see that the proposed analytical result (UniPLAN) performs closely $(<5 \%$ degradation) to the optimal conflict radius (UniOPT) derived from the exhaustive search. This result again demonstrates the importance of optimizing the conflict graph - A $5 \mathrm{~m}$ error from the optimal $r$ can lead to at least $20 \%$ drop in the spectrum utilization. Further, the worst-case analysis UniCSV suffers from $35 \%$ degradation in the spectrum utilization by over-estimating the amount of interference. Finally, we examine the impact of various network parameters on UniPLAN in Fig. 8. Similar conclusions can be drawn from these results.

\section{The Gain of Local Adjustments}

We now examine the performance of PLAN that starts from the analytically derived $r^{*}$ and then refines the solution using the iterative local adjustments. Our results are averaged over 100 uniform topologies using $R=300 \mathrm{~m}$ and $D=30 \mathrm{~m}$.

Fig. 9a compares PLAN and UniPLAN by examining their performance distance to UniOPT. We can see that the first step of PLAN (i.e. UniPLAN) can achieve over $95 \%$ of the UniOPT's utilization. With additional local adjustments, PLAN outperforms UniOPT by up to $15 \%$. From the cumulative distributed function (CDF) of the received SINR in Fig. 9b, we see that PLAN effectively reduces the number of nodes with high SINRs while ensuring all the transmissions have SINR higher than $\beta$. 


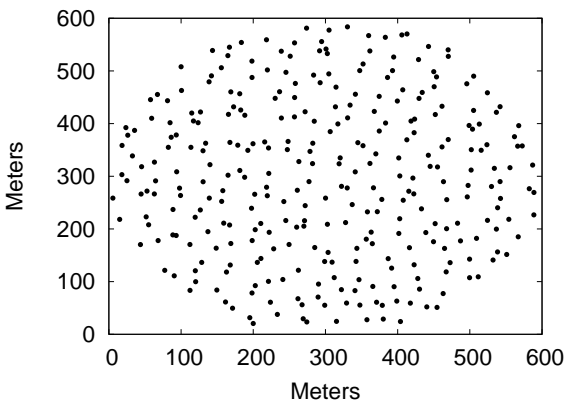

(a) Sample Uniform Topology

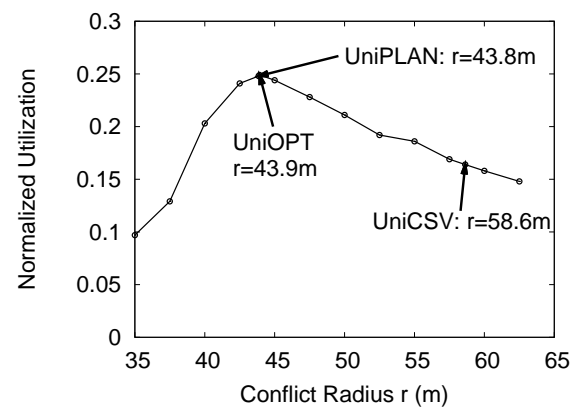

(b) $\alpha=2, d=5 \mathrm{~m}$

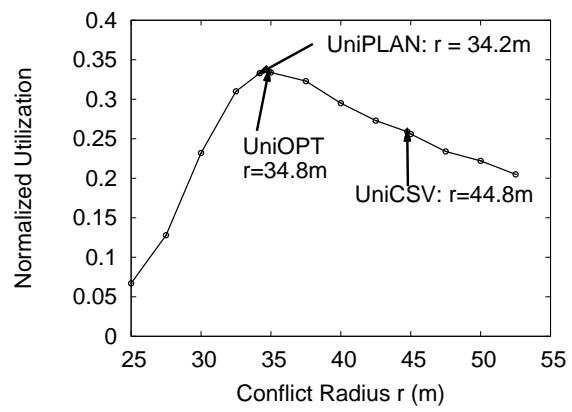

(c) $\alpha=3, d=10 \mathrm{~m}$

Fig. 7. The performance of UniPLAN in a uniform circular topology with $R=300 \mathrm{~m}$ and $D=30 \mathrm{~m}$.

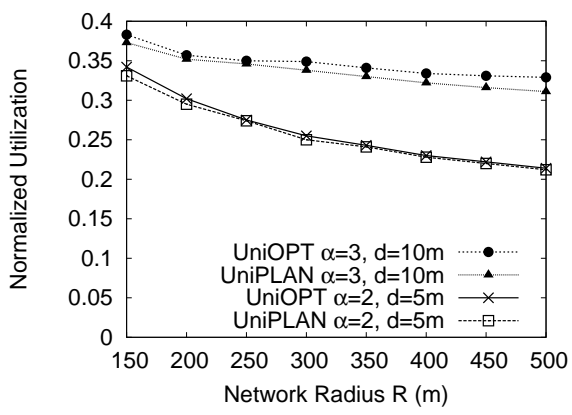

(a) Impact of $\mathrm{R}$

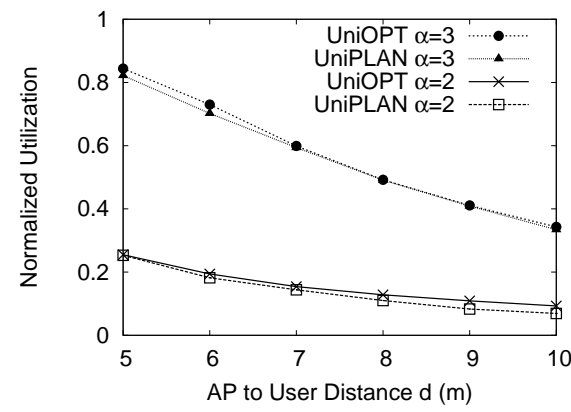

(b) Impact of $\mathrm{d}$

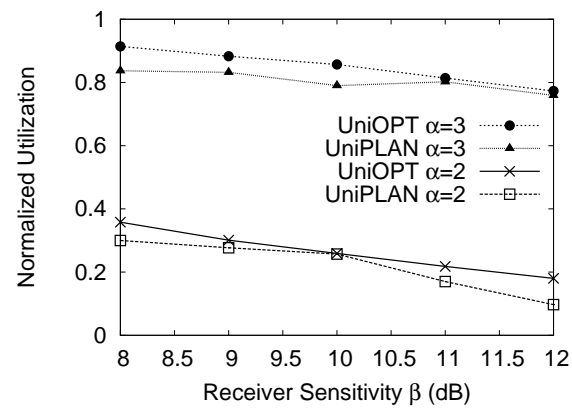

(c) Impact of $\beta$

Fig. 8. The impact of various parameters on UniPLAN in uniform circular topologies, cell size $D=30 \mathrm{~m}$. Each data point in the figures is averaged by 10 topologies using the same settings.

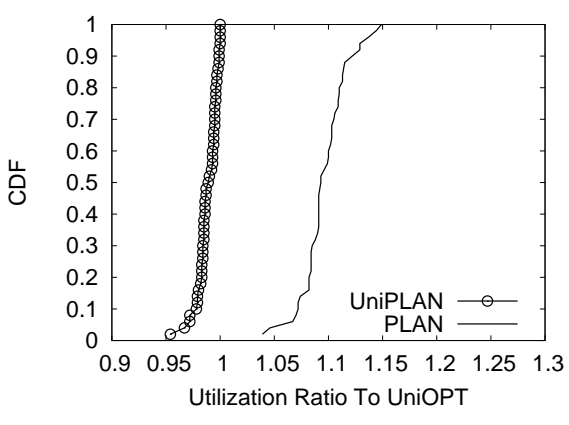

(a) UniPLAN and PLAN v. UniOPT

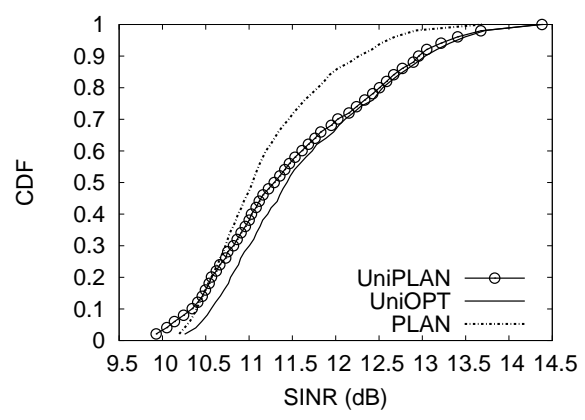

(b) CDF of SINR

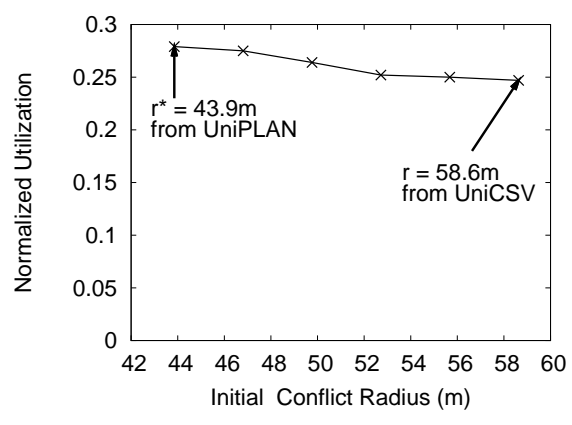

(c) Choosing Initial $r$

Fig. 9. The gain of PLAN's local adjustments in uniform circular topologies, network radius $R=300 \mathrm{~m}$, cell size $D=30 \mathrm{~m}$, propagation exponent $\alpha=2$.

In PLAN, one design decision is to start the local adjustments from an initial conflict graph produced with $r^{*}$. We justify this choice by evaluating the performance of PLAN using different initial conflict graphs. These conflict graphs are produced using different $r$. Results in Fig. 9c show that the use of $r^{*}$ in the initial conflict graph leads to the best performance. This is because that the performance of UniPLAN is very close to the optimal solution, and starting from such conflict graph can effectively reduce the number of nodes that fall into a local maxima state during the adjustments.

Even in uniform networks, we see that PLAN outperforms UniOPT. This is because the local adjustments help balance the distribution of SINR across active transmissions. However, in these topologies, the improvement is relatively small, because the uniform topologies lead to almost uniform interference conditions.

\section{Impact of Network Topology}

We now examine the performance of PLAN under different network topologies. We start from the clustered topologies. Fig. 10a illustrates a sample topology where 300 nodes are placed in a $600 \mathrm{~m}$ by $600 \mathrm{~m}$ square region with one dense hotspot $^{\S}$. We simulate 100 clustered topologies and compare

\footnotetext{
$\S$ To derive the conflict radius $r^{*}$ of UniPLAN, we set $R=300 \mathrm{~m}$ in (14)
} 


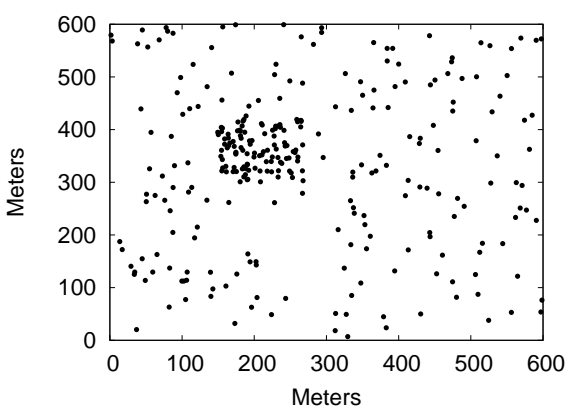

(a) Sample Clustered Topology

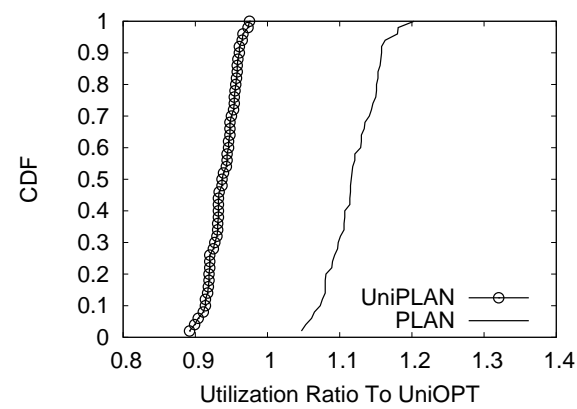

(b) Compare Normalized Utilization to UniOPT

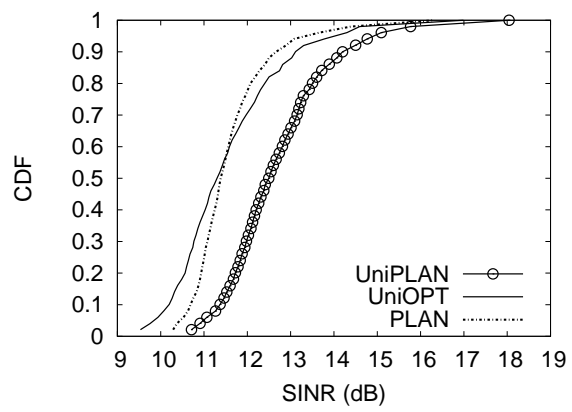

(c) CDF of SINR

Fig. 10. The performance of PLAN in clustered topologies with 300 nodes in a $600 \mathrm{~m}$ by $600 \mathrm{~m}$ square region, propagation exponent $\alpha=2$.

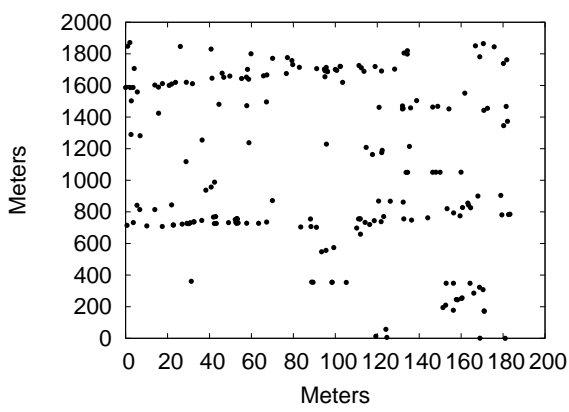

(a) Sample Measured Topology

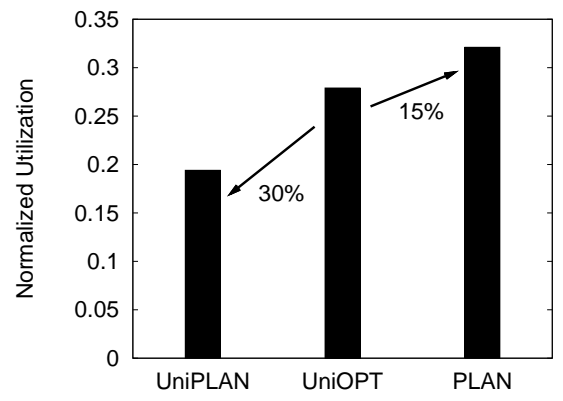

(b) Normalized Utilization

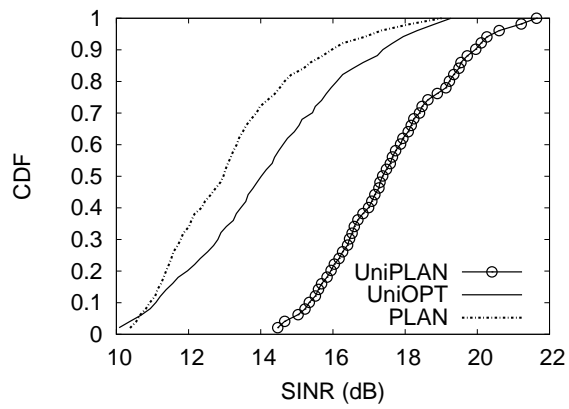

(c) $\mathrm{CDF}$ of SINR

Fig. 11. The performance of PLAN in a measured AP trace from PlaceLab [10], propagation exponent $\alpha=2$.

the normalized utilization of UniPLAN and PLAN over that of UniOPT, shown in Fig. 10b in terms of the CDF of the ratios. We see that UniPLAN achieves over $90 \%$ of the utilization from UniOPT, and PLAN outperforms UniOPT by up to $20 \%$. These results show that although the clustered topology does not meet the analytical assumptions, our analytical estimation UniPLAN still provides good performance. Furthermore, for clustered topologies with highly heterogenous interference conditions, PLAN's location adjustments provide great benefits.

Next, we examine the performance of PLAN using measured AP topologies [10]. Fig. 11a illustrates a sample topology where 200 nodes are distributed in a $200 \mathrm{~m}$ by $2000 \mathrm{~m}$ rectangular region, and the node placement is highly irregular. We assign each node one user with distance $d=5 \mathrm{~m}$. We use $R=1000 \mathrm{~m}$ to estimate $r^{*}$ in UniPLAN. Fig. $11 \mathrm{~b}$ and c compare the normalized utilization and the CDF of the received SINR of UniPLAN, UniOPT and PLAN, respectively. We see that PLAN outperforms UniOPT by $15 \%$ by locally adapting individual conflict radius to heterogeneous interference conditions. We also see that UniPLAN results in $30 \%$ performance degradation compared to UniOPT. This is partially because the computations of UniPLAN assumes a circular area, and the use of $R=1000 \mathrm{~m}$ overestimates the interference and produces highly conservative conflict graph/allocation. This can be seen from Fig. 11c where the SINRs are much higher than $\beta$.

\section{E. PLAN vs. PhyOPT}

Finally, we compare PLAN to PhyOPT, which produces the optimal spectrum allocation directly from the physical interference model. We derive PhyOPT using the exhaustive search. Because of its computational complexity, we use small networks of 30 nodes in a $150 \mathrm{~m}$ by $150 \mathrm{~m}$ square region. Our results are averaged over 50 random deployments. Fig. 12 shows the performance degradation of UniPLAN, UniOPT and PLAN when compared to PhyOPT. We see that PLAN achieves less than $20 \%$ performance degradation to PhyOPT, which demonstrates its efficiency. The difference between UniPLAN and PLAN again demonstrates the critical need of the local adjustments.

\section{Discussions}

\section{A. Practical Physical Environments}

In this paper, we analyze and evaluate PLAN under simplified assumptions on signal propagation and radio hardware. For example, we calculate the received interference/signal power using the Geometry pathloss equation $P_{R X}=P_{T X} / d^{\alpha}$, and use the $\beta$ threshold model to capture the receiver performance. Some of these assumptions may not hold in practical scenarios. For example, the receiver signal and interference strength may fluctuate because of channel fading and shadowing; the receiver bit error rate is a continuous function of the received SINR unless using a capacity-achieving coding; and the SINR measurements may contain errors. 


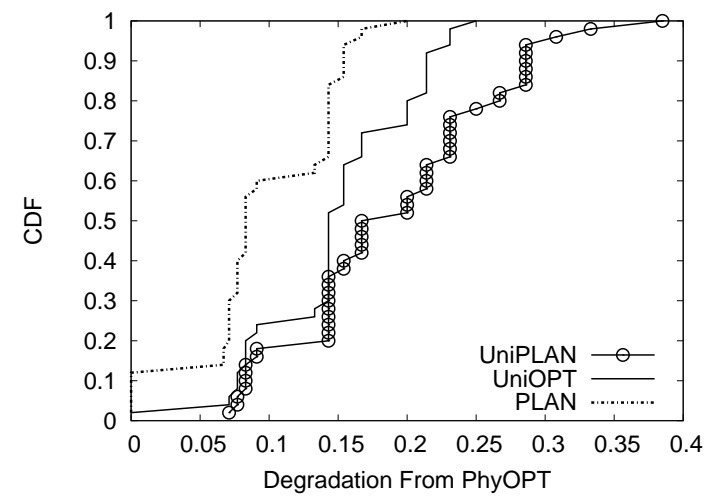

Fig. 12. Comparing PLAN, UniPLAN to PhyOPT, the global optimal allocation derived from the physical interference models; $\alpha=2$. The results are shown in terms of the degradation in utilization over that of PhyOPT.

Using the same methodology, the design of PLAN can be extended to account for these practical issues. First, note that PLAN estimates the optimal conflict radius by estimating the level of cumulative interference and then defining a proper conflict region to guarantee the receiver SINR beyond $\beta$. We can integrate sophisticated radio propagation models into this process. For example, if the transmission experiences the Rician fading, we can use $\beta+\Delta \beta$ to estimate $r^{*}$ where $\Delta \beta$ is a fading compensation factor. We plan to address this issue in a future paper.

Second, even without a good model of practical environments, PLAN applies reactive local adjustments to improve its conflict detection and spectrum allocation based on each node's observation of environment. The basic idea remains the same: for nodes who observe undesirable SINR, we increase the number of their conflict neighbors to reduce interference; and for nodes who observe excessively high SINR, we decrease the number of their conflict neighbors to increase the spatial reuse. Note that the environment observation can come from not only the SINR measurements but also the throughput or packet error rate measurements. Therefore, PLAN can make the allocation adjustments using a commodity radio hardware.

\section{B. Integrating Power Control}

Power control has been proposed to control network topology, increase spacial reuse [2] and reduce interference. Intuitively, PLAN can be integrated with power control. For downlink transmissions (AP to user), APs can first transmit at the highest power and then apply PLAN and a channel allocation mechanism to assign conflicting transmissions to different channels. When the allocation result cannot support all the transmissions, APs apply power control to reduce the level of interference. The system then reapplies PLAN and the channel allocation mechanism to determine the new allocation. Together, power control/PLAN/channel allocation can be applied iteratively to optimize the spectrum usage. The fundamental challenge is how to jointly optimize power control and channel allocation.

\section{RELATED WORK}

The most relevant work in literatures can be classified into three categories.

As stated earlier, there are a number of initial proposals for generating conflict graphs. [13] proposed to use the degradation of throughput to measure the interference between two transmission links. [15] improved the scalability of this method through theoretical modelling based on RSSI measurements. [1] proposed to use probes to measure whether two transmissions conflict. These approaches infer conflict relationships based only on pairwise interference characteristics, such as signal strength or throughput. In this paper we propose that this effort must explicitly take into account the accumulative effect of interference in order to optimize the allocation. In particular, we proposed an analytical framework to derive the uniform conflict radius, which captures the impact of cumulative interference and leads to significant improvements in the spectrum utilization.

The spectrum/channel allocation problem under the graph model has been widely studied in existing literatures. Among the most relevant ones, [14] designed centralized algorithms based on a variation of graph coloring model. [5], [22] proposed low-complexity algorithms based on local adaptation. [16] proposed algorithms that optimize channel allocation by considering APs' traffic demands. [3] designed centralized algorithms to assign spectrum blocks that are contiguous in frequency. Our allocation-aware analytical framework in this paper can be used in conjunction with the above algorithms and many others to optimize the spectrum allocation.

Finally, the difference between the physical interference model and the graph model is an interesting problem. Recent literatures have found fundamental differences between the two in terms of wireless capacity and link scheduling efficiency [6], [12]. In this paper, we found somewhat surprisingly that the graph model can achieve the same optimal result as the physical model in terms of spectrum allocation. Our results validate the use of graph-based interference models as a reasonable approximation for the physical model. It would also be interesting to see whether the technique used in the proof of Theorem 1 can be applied to the wireless capacity and link scheduling problems.

\section{CONCLUSIONS}

In this paper, we propose PLAN, a systematic framework to produce conflict graphs to capture the complex characteristics of radio interference. PLAN combines a proactive estimation with a reactive adjustment mechanism. First, assuming uniform interference conditions, PLAN applies an analytical framework to define the criterion for detecting conflicting neighbors, integrating the cumulative effect of interference and the impact of spectrum allocation algorithms. Second, PLAN applies a local adjustment algorithm to refine the conflict criterion at individual nodes based on their observed performance, addressing inherent heterogeneity in interference conditions. Through detailed analysis and experimental evaluation, we 
show that PLAN effectively converts the complex interference conditions into a proper conflict graph, enabling us to reuse many existing conflict-graph based spectrum allocation solutions. While the current design of PLAN considers ideal propagation conditions, our future work is to integrate practical issues such as fading, hardware characteristics and power settings, and implement and evaluate PLAN using physical radio testbeds.

\section{REFERENCES}

[1] Ahmed, N., And Keshav, S. Smarta: A self-managing architecture for thin access points. In In Proc. of CoNext 2006.

[2] Akella, A., Judd, G., Seshan, S., and Steenkiste, P. Selfmanagement in chaotic wireless deployments. In Proc. of Mobicom 2005.

[3] Bahl, P., Chandra, R., Moscibroda, T., Wu, Y., and Yuan, Y. Load aware channel-width assignments in wireless lans. Tech. Rep. 2007-79, Microsoft Research, 2007.

[4] Brar, G., Blough, D. M., And S Anti, P. Computationally efficient scheduling with the physical interference model for throughput improvement in wireless mesh networks. In Proc. of MobiCom (2006).

[5] CAO, L., AND Zheng, H. Spectrum allocation in ad hoc networks via local bargaining. In Proc. of SECON (2005).

[6] Gronkvist, J., AND Hansson, A. Comparison between graph-based and interference-based stdma scheduling. In Proc. of MobiHoc (2001).

[7] Gupta, P., AND Kumar, P. R. The capacity of wireless networks. IEEE Transactions on Information Theory 46, 2 (2000), 388-404.

[8] Jain, K., Padhye, J., Padmanabhan, V., and Qiu, L. Impact of interference on multi-hop wireless network performance. In Proc. of MobiCom (2003).

[9] KIM, T.-S., Lim, H., AND Hou, J. C. Improving spatial reuse through tuning transmit power, carrier sense threshold, and data rate in multihop wireless networks. In Proc. of MobiCom (2006).

[10] LaMarca, A., ET AL. Place lab: Device positioning using radio beacons in the wild. In Proc. of Pervasive (June 2005).

[11] Mishra, A., Shrivastava, V., Agarwal, D., BanerJee, S., And GANGULY, S. Distributed channel management in uncoordinated wireless environments. In Proc. of MobiCom (2006).

[12] Moscibroda, T., Wattenhofer, R., And Weber, Y. Protocol Design Beyond Graph-Based Models. In 5th Workshop on Hot Topics in Networks (HotNets), Irvine, California, USA (2006).

[13] Padhye, J., Agarwal, S., Padmanabhan, V. N., Qiu, L., And RAO, A. Estimation of link interference in static multi-hop wireless networks. In Proc. of IMC (Oct. 2005).

[14] Peng, C., Zheng, H., And Zhao, B. Y. Utilization and fairness in spectrum assignemnt for opportunistic spectrum access. Mobile Networks and Applications (MONET) 11 (May 2006), 555-576.

[15] Reis, C., Mahajan, R., Rodrig, M., Wetherall, D., and ZaHORJAN, J. Measurement-based models of delivery and interference in static wireless networks. In Proc. of SIGCOMM (2006).

[16] Rozner, E., Mehta, Y., AKella, A., And QiU, L. Traffic-aware channel assignment in enterprie wireless networks. In Proc. of ICNP (2007).

[17] То́тн, L. F. On the densest packing of circles in a convex domain. Norske Vid. Selsk. Fordhl., Trondheim 21 (1948), 68-76.

[18] TURÁN, P. On an extremal problem in graph theory (in hungarian). Mat. Fiz. Lapok 48 (1941), 436-452. English translation in Collected papers of Paul Turán (P.Edrös, Ed.), 231-240, Akadémiai Kiadó, Budapest, 1990.

[19] Xing, K., Cheng, X., Ma, L., , And Liang, Q. Superimposed code based channel assignment in multi-radio multi-channel wireless mesh networks. In Proc. of MobiCom (2007).

[20] YANG, X., AND VAIDYA, N. H. On the physical carrier sense in wireless ad hoc networks. In Proc. of INFOCOM (2005).

[21] Yuan, Y., Bahl, P., Chandra, R., Moscibroda, T., Narlanka, S., AND WU, Y. Allocating dynamic time-spectrum blocks in cognitive radio networks. In Proc. of MobiHoc (2007).

[22] Zheng, H., AND CAO, L. Device-centric spectrum management. In Proc. of IEEE DySPAN (November 2005). 